\title{
Ab Initio Approach and Its Impact on Superconductivity
}

\author{
Defang Duan ${ }^{1,2}$ (D) Hongyu Yu ${ }^{1} \cdot$ Hui Xie $^{1} \cdot$ Tian Cui $^{1}$
}

Received: 19 September 2018 / Accepted: 24 September 2018 / Published online: 24 October 2018

(c) The Author(s) 2018

\begin{abstract}
One of the main motivations for studying superconductivity is to search for high-temperature superconductors, especially room-temperature superconductors. During the long history of more than 100 years since the discovery of superconductivity, a number of high-temperature superconductors were found and several great breakthroughs were achieved. Recently, thanks to advances in computing power, progress in crystal structure prediction, and developments in density functional theory, computations have been carried out to predict the structures and an appearance of superconductivity of hydrides at high pressure. More exciting, it is been the first time when a simple, perfect cubic phase of $\mathrm{H}_{3} \mathrm{~S}$ which become superconductor at $T_{\mathrm{c}}=200 \mathrm{~K}$ under high pressure was successfully predicted by means of ab initio calculation, and then confirmed experimentally. This observation breaks the temperature record of cuprate with $T_{\mathrm{c}}=164 \mathrm{~K}$ and further stimulates the studies of hydrides under pressure. Very recently, the high value of $T_{\mathrm{c}}=286 \mathrm{~K}$ was theoretically predicted for LaH 10 at $210 \mathrm{GPa}$ and this prediction has been confirmed experimentally. These two successful examples demonstrate the importance of ab initio approach to superconductivity.
\end{abstract}

Keywords Ab initio calculations $\cdot$ Superconductivity $\cdot$ High-pressure $\cdot$ Hydrides

\section{Progress in the Field of Superconductivity}

Ever since Onnes [1] discovered the phenomenon of superconductivity in 1911, searching for high-temperature superconductors (see Fig. 1) and understanding the mechanism of such a behavior have become one of the hot spots in the field of condensed matter physics. Initially people searched superconductors mainly among the materials containing transition metal (TM) with superconducting transition temperatures $\left(T_{\mathrm{c}}\right)$ 17-23 K. For example, wellknown materials with the A15 structure $\left(\mathrm{Nb}_{3} \mathrm{Sn}\right.$ and $\mathrm{Nb}_{3} \mathrm{Ge}$ [2]) were studied. In 1986, J. Bednorz and K. Müller discovered a superconductivity in the $\mathrm{Ba}-\mathrm{La}-\mathrm{Cu}-\mathrm{O}$ system with $T_{\mathrm{c}}=$

Defang Duan

duandf@jlu.edu.cn

Tian Cui

cuitian@jlu.edu.cn

1 State Key Laboratory of Superhard Materials, College of Physics, Jilin University, Changchun 130012, China

2 Department of Materials Science and Metallurgy, University of Cambridge, 27 Charles Babbage Road, Cambridge CB3 OFS, UK
35 K [3]. Later, more cuprate superconductors were discovered, and the critical temperature recorded was constantly increased. For example, the $T_{\mathrm{c}}$ of the $\mathrm{Hg}-\mathrm{Ba}-\mathrm{Ca}-\mathrm{Cu}-\mathrm{O}$ system was measured to be $133 \mathrm{~K}$ [4] at normal pressure and reached $164 \mathrm{~K}$ at about $31 \mathrm{GPa}$ [5]. Most of physicists have long believed that if magnetic elements (such as iron and nickel) are added to the superconductors, the superconductivity will be destroyed. However, discovery of the first iron-based superconductor La-Fe-As-O system with $T_{\mathrm{c}}=$ $26 \mathrm{~K}$ drastically changed the views of scientists. In the following years, new iron-based superconductors such as iron arsenide and iron selenide were continuously discovered. At high pressure, the highest $T_{\mathrm{c}}$ of samarium-doped $\mathrm{SrFeAsF}$ had been reached $56 \mathrm{~K}$ [6]. Moreover, the study on singlelayer films of FeSe which grown on a $\mathrm{Nb}$-doped $\mathrm{SrTiO}_{3}$ substrate showed a $T_{\mathrm{c}}$ as high as $109 \mathrm{~K}$ due to the interface effects [7]. However, the progress of traditional superconductors is relatively slow. In 2001, Nagamatsu et al. [8] found the bulk superconductivity in $\mathrm{MgB}_{2}$ by magnetization and resistivity measurements and reported the $T_{\mathrm{c}}$ of $\mathrm{MgB}_{2}$ is $39 \mathrm{~K}$. Although the $T_{\mathrm{c}}$ of $\mathrm{MgB}_{2}$ is much lower than that of copper oxide superconductors, $\mathrm{MgB}_{2}$ is relatively inexpensive and easy to synthesize, so it means that the $\mathrm{MgB}_{2}$ has the conditions for scaled production and applications. 
Fig. 1 Discovery time of superconductors and critical temperature

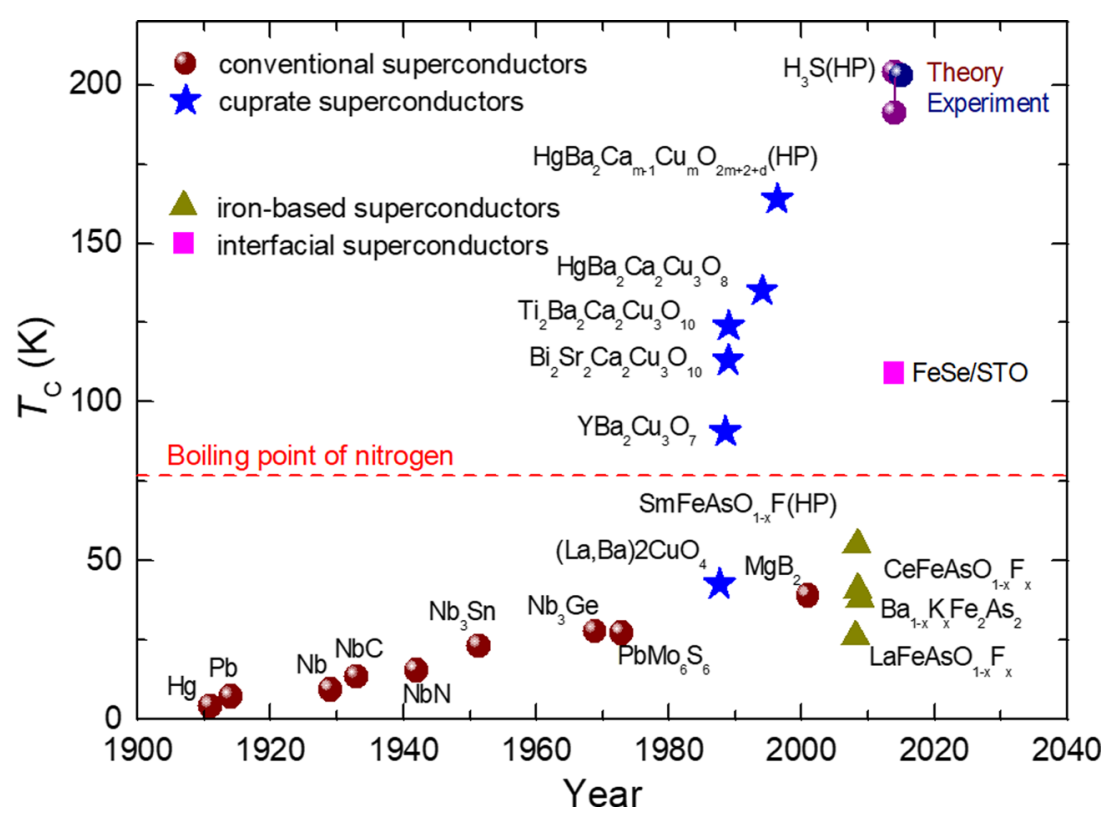

\section{Ab Initio Calculation on Superconductivity and Structure Prediction}

In the 1970s and 1980s, quantitative prediction of electronic structures based on density functional theory (DFT) was developed. But, the values of the vibrational frequencies of phonon were usually taken from experiment. By the 1990s, phonons could be computed accurately from ab initio calculation and matrix elements can be calculated from linear response theory. In the twenty-first century, electronphonon coupling (EPC) could be computed for materials from ab initio calculation and this allows us to estimate superconducting transition temperature.

The EPC spectral function $\alpha^{2} F(\omega)[9,10]$ can be evaluated from the phonon linewidth $\gamma_{q j}$ in the first Brillouin zone (BZ).

$\alpha^{2} F(\omega)=\frac{1}{2 \pi N\left(\varepsilon_{\mathrm{F}}\right)} \sum_{q j} \frac{\gamma_{q j}}{\omega_{q j}} \delta\left(\omega-\omega_{q j}\right) w(q)$

where $\omega_{q j}$ is the mode frequency and $N\left(\varepsilon_{\mathrm{F}}\right)$ is the density of states at the Fermi level $\left(\varepsilon_{\mathrm{F}}\right) \cdot \gamma_{q j}$, the linewidth of a phonon mode $j$ at wave vector $q$, is given by

$$
\begin{aligned}
\gamma_{q j}= & 2 \pi \omega_{q j} \sum_{n m} \int \frac{d^{3} k}{\Omega_{\mathrm{BZ}}}\left|g_{k n, k+q m}^{j}\right|^{2} \delta\left(\varepsilon_{k n}-\varepsilon_{\mathrm{F}}\right) \delta \\
& \times\left(\varepsilon_{k+q m}-\varepsilon_{\mathrm{F}}\right)
\end{aligned}
$$

where the integral is over the first $\mathrm{BZ}$ and $\varepsilon_{k n}$ and $\varepsilon_{k+q m}$ are the Kohn-Sham eigenvalues with wave vectors $k$ and $k+q$ within the $n$th and $m$ th bands, respectively. $g_{k n, k+q m}^{j}$ is the electron-phonon matrix element that determined from the linearized self-consistent potential. The $\gamma_{q j}$ is related to the mode EPC parameter $\lambda_{q j}$ by

$\lambda_{q j}=\frac{\gamma_{q j}}{\pi \hbar N\left(\varepsilon_{\mathrm{F}}\right) \omega_{q j}^{2}}$

The EPC parameter $\lambda$ can be given by

$\lambda=2 \int \frac{\alpha^{2} F(\omega)}{\omega} d \omega \approx \sum_{q j} \lambda_{q j} \omega(q)$

where $\omega(q)$ is the weight of a $q$ point in the first BZ. The superconducting transition temperature $\left(T_{\mathrm{c}}\right)$ can be estimated with the Allen-Dynes-modified McMillan equation $[11,12]$ as follows:

$T_{\mathrm{c}}=\frac{\omega_{\log }}{1.2} \exp \left[-\frac{1.04(1+\lambda)}{\lambda-\mu^{*}(1+0.62 \lambda)}\right]$

where $\omega_{\log }$ is the logarithmic average frequency and $\mu^{*}$ is the Coulomb pseudopotential. Equation (5) is found to be accurate for materials with $\lambda<1.5$. When $\lambda>1.5$, two separate correction factors $\left(f_{1}\right.$ and $\left.f_{2}\right)$ are introduced [11]:

$T_{\mathrm{c}}=\frac{f_{1} f_{2} \omega_{\log }}{1.2} \exp \left[-\frac{1.04(1+\lambda)}{\lambda-\mu^{*}(1+0.62 \lambda)}\right]$

where $f_{1}=\left[1+\left(\frac{\lambda}{\Lambda_{1}}\right)^{\frac{3}{2}}\right]^{\frac{1}{3}}$ and $f_{2}=1+\frac{\left(\frac{\bar{\omega}_{2}}{\omega_{\log }}-1\right) \lambda^{2}}{\lambda^{2}+\Lambda_{2}^{2}}$ and $\Lambda_{1}=2.46\left(1+3.8 \mu^{*}\right)$ and $\Lambda_{2}=1.82\left(1+6.3 \mu^{*}\right) \frac{\bar{\omega}_{2}}{\omega_{\log }}$ and $\bar{\omega}_{2}=<\omega^{2}>^{\frac{1}{2}}$ and $<\omega^{2}>=\frac{2}{\lambda} \int d \omega \alpha^{2} F(\omega) \omega$.

In addition, the superconducting state of a material normally is very sensitive to its structure. Therefore, it is very important to know the arrangement of the atoms in a material and determine the correct structures. However, 
finding the thermodynamically most stable structure at a given temperature and pressure with the only known information of chemical compositions is extremely difficult as it basically involves a huge number of energy minima in the potential energy surface (PES). Owing to the spectacular advances in computer hardware coupled with the developments in DFT, it is now possible to predict the crystal structure using the quantum mechanical methods. Recently, a number of different approaches to search for stable or metastable structures of materials based on the chemical composition and the external conditions with the use of the first-principles electronic structure methods have been developed, such as minima hopping algorithm [13], evolutionary/genetic algorithms [14, 15], random sampling algorithm [16], and swarm intelligence [17]. The various searching methods exhibit many similar features and are successful in many applications. The minima hopping algorithm need the input of initially guessed structure. If the initial structure on PES is well chosen, it will converge fast into the global minimum. The evolutionary/genetic algorithms, random sampling algorithm, and swarm intelligence implemented in several efficient software packages (e.g., USPEX, CALYPSO, AIRSS, and XtalOpt) are unbiased methods, which are not relying on any initial guess of structures.

\section{Superconducting Hydrides}

According to the Bardeen-Cooper-Schrieffer (BCS) theory, the superconducting transition temperature $\left(T_{\mathrm{c}}\right)$ is directly proportional to Debye temperature, while its value is inversely proportional to the mass of element. This tells us that we should search high-temperature superconductors among the light element materials. Hydrogen is the lightest element, and its Debye temperature can reach $3500 \mathrm{~K}$. However, it is well known that solid hydrogen is an insulator at ambient conditions. It has been predicted for a long time that high pressure can effectively reduce the band gap and make the transition of the insulating hydrogen into a metallic state, that is into a metallic hydrogen atom. As a result, searching for a metallic phase of solid hydrogen has become a very important topic in physics and related fields. Metallic hydrogen also becomes one of the best candidates for room-temperature superconductor. Last year, signs of metallic hydrogen were observed at about $495 \mathrm{GPa}$ [18], but further evidence are needed [19]. So, up to now, there is no conclusive proof for getting the metallic hydrogen.

Recently, parallel to the metallic hydrogen research, hydrogendominated materials at pressures are getting more and more important It has become a hot topic for searching high-temperature superconductors. The idea has been proposed by Ashcroft [20] 10 years ago. Hydrogendominated materials can be metallized at much lower pressures due to the presence of the non-hydrogen element. Because such materials are dominated by hydrogen elements, important novel properties can be found after metallization, such as high $T_{\mathrm{c}}$ superconductivity.

In situ diamond anvil cell (DAC) experimental studies of the metallization and superconductivity of the hydrogendominated materials at very high pressure represent a serious challenge. Fortunately, the DFT calculations are relatively cheap and improvements in accuracy have motivated ambitious studies in superconductivity of the materials dominated by the presence of hydrogen. Therefore, theoretical researches have been at the forefront of this field, motivated, aided, or guided many experimental studies and made outstanding contributions. One of the most exciting new achievements is the remarkable value of $T_{\mathrm{c}}=191-204 \mathrm{~K}$ for $I m-3 m \mathrm{H}_{3} \mathrm{~S}$ at $200 \mathrm{GPa}$ firstly predicted by ab initio calculation [21]. Then, experiment measurement [22] discovered critical temperatures up to $T_{\mathrm{c}}=203 \mathrm{~K}$ at $155 \mathrm{GPa}$ in compression of hydrogen sulfide which perfectly consistent with the theoretical prediction. The high $T_{\mathrm{c}}$ exceeds the lowest temperature recorded on Earth at the ground level of $184 \mathrm{~K}$ and breaks the previous record of $164 \mathrm{~K}$ in cuprate superconductors. Experimental confirmation of this prediction demonstrated the remarkable power of ab initio calculation. Yet the achievement was not produced unexpectedly by sheer good luck, but it represents the result of the long-term exploration of hydrogendominated materials by numerous researchers. For example, an experimental study found $\mathrm{SiH}_{4}[23,24]$ metallized at $50-60 \mathrm{GPa}$, and the highest $T_{\mathrm{c}}$ was $17.5 \mathrm{~K}$ at $96 \mathrm{GPa}$. The theoretical studies suggested that the $T_{\mathrm{c}}$ of $\mathrm{SiH}_{4}[25,26], \mathrm{GeH}_{4}[27,28]$, $\mathrm{SnH}_{4}[29,30], \mathrm{Si}_{2} \mathrm{H}_{6}$ [31] , and $\mathrm{B}_{2} \mathrm{H}_{6}$ [32] at high pressure is $16 \sim 106 \mathrm{~K}, 40 \sim 64 \mathrm{~K}, 62 \sim 80 \mathrm{~K}$, and $139 \mathrm{~K}$; the highest $T_{\mathrm{c}}$ of new hydrides $\mathrm{SiH}_{4}\left(\mathrm{H}_{2}\right)_{2}$ [33], $\mathrm{GeH}_{4}\left(\mathrm{H}_{2}\right)_{2}$ [34], $\mathrm{KH}_{6}$ [35], and $\mathrm{CaH}_{6}$ [36] under high pressure is $107 \mathrm{~K}, 90 \mathrm{~K}, 80$ $\mathrm{K}$, and $235 \mathrm{~K}$, respectively.

\section{Superconductivity in Sulfur Hydrides}

In 2011, Strobel et al. [37] reported that mixing $\mathrm{H}_{2} \mathrm{~S}$ and $\mathrm{H}_{2}$ can form a stoichiometric compound $\left(\mathrm{H}_{2} \mathrm{~S}\right)_{2} \mathrm{H}_{2}\left(\mathrm{H}_{3} \mathrm{~S}\right.$, the stoichiometric ratio of $\mathrm{H}$ and $\mathrm{S}$ atoms is $3: 1$ ) at about $3.5 \mathrm{GPa}$ and the highest pressure is just only $30 \mathrm{GPa}$. In 2014, structure calculations with the predictions for stoichiometric compound $\mathrm{H}_{3} \mathrm{~S}$ in the pressure range 20-300 GPa were performed [21] using an evolutionary algorithm implemented in the USPEX code. The ab initio calculation shows that the $P 1$ structure (see Fig. $2 a$ ) is the most stable structure below $37 \mathrm{GPa}$. With the increase of pressure, the hydrogen bond interaction gradually increased and formed the orthogonal $\mathrm{Cccm}$ structure (37-111 GPa; see Fig. 2b). 
Fig. 2 High-pressure crystal structures of $\mathrm{H}_{3} \mathrm{~S}$. a $P 1$ structure. b $C c c m$ structure. c $R 3 m$ structure. d $I m-3 m$ structure. Large spheres represent $\mathrm{S}$ atoms, and small spheres denote $\mathrm{H}$ atoms, respectively

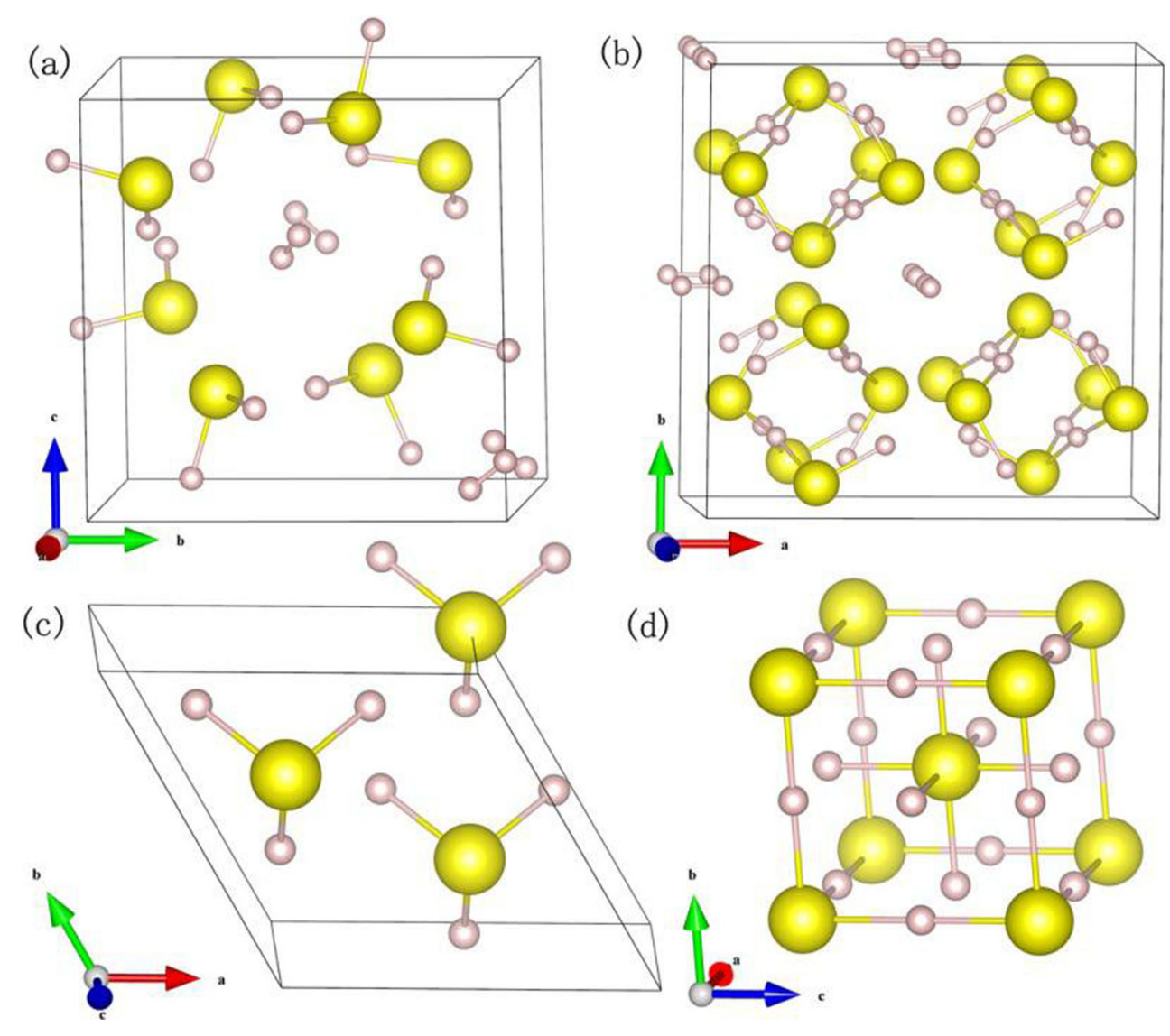

Both $P 1$ and $C c c m$ structures contain $\mathrm{H}_{2} \mathrm{~S}$ and $\mathrm{H}_{2}$ molecular units and form a $\left(\mathrm{H}_{2} \mathrm{~S}\right)_{2} \mathrm{H}_{2}$ "host-guest" structure. Under higher pressure, the $\mathrm{Cccm}$ structure transforms to a trigonal structure with $R 3 m$ symmetry, as shown in Fig. 2c. With the further increase in pressure, the cubic $I m-3 m$ structure (Fig. 2d) become more stable, in which the hydrogen bond is symmetrization completely. In the $R 3 \mathrm{~m}$ and $I m-3 m$ structures, both $\mathrm{H}_{2} \mathrm{~S}$ and $\mathrm{H}_{2}$ molecular units have disappeared and $\mathrm{H}-\mathrm{S}$ bonds have metallic covalent characteristic. Further calculation shown that the electron coupling parameter $(\lambda)$ and superconducting transition temperature $\left(T_{\mathrm{c}}\right)$ of the $R 3 \mathrm{~m}$ structure are 2.07 and $155 \sim 166 \mathrm{~K}$ with $\mu^{*}=0.1-0.13$ at $130 \mathrm{GPa}$, respectively. More importantly, the calculated $\lambda$ and $T_{\mathrm{c}}$ for the $I m-3 m$ $\mathrm{H}_{3} \mathrm{~S}$ at $200 \mathrm{GPa}$ reach high values of 2.19 and 191 204 $\mathrm{K}\left(\mu^{*}=0.1-0.13\right)$, respectively. It is shown that for $R 3 m$ phase, $T_{\mathrm{c}}$ increases with increasing pressure, while for Im$3 \mathrm{~m}$ phase, $T_{\mathrm{c}}$ decreases with increasing pressure. This is the first time the crystal structures and $\operatorname{high} T_{\mathrm{c}}$ superconductivity of $\mathrm{H}_{3} \mathrm{~S}$ at high pressure were proposed.

Later, Drozdov et al. [38] published a reprint paper on arXiv reported based on electrical measurements, that compressed the $\mathrm{H}_{2} \mathrm{~S}$ sample transformed to superconductor with $T_{\mathrm{c}}=150 \mathrm{~K}$ at about $200 \mathrm{GPa}$. One can see that $T_{\mathrm{c}}$ steadily increased with pressure in the regime of $107^{\sim} 160$ $\mathrm{GPa}$. This is in general agreement with Li et al's calculations of $T_{\mathrm{c}}$ for $\mathrm{H}_{2} \mathrm{~S}$ [39]. Remarkably, in the second stage, $T_{\mathrm{c}}$ increased sharply to $190 \mathrm{~K}$ at about $155 \mathrm{GPa}$ and it decreased with increasing pressure. The value of $T_{\mathrm{c}}$ and its dependence on pressure in the experiment (Fig. 3) are extremely identical with Duan et al's predicted $\mathrm{Im}-3 m-\mathrm{H}_{3} \mathrm{~S}$ phase [21]. Therefore, it is speculated that $\mathrm{H}_{2} \mathrm{~S}$ samples have decomposed to $\mathrm{H}_{3} \mathrm{~S}$ crystal under high pressure and the high $T_{\mathrm{c}}$ comes from theoretically predicted $I m-3 m-\mathrm{H}_{3} \mathrm{~S}$ [21]. Furthermore, theoretical researches [40, 41] showed that $\mathrm{H}_{2} \mathrm{~S}$ does decomposed into $\mathrm{S}$ and new compounds $\mathrm{H}_{3} \mathrm{~S}$ at high pressure and $\mathrm{H}_{3} \mathrm{~S}$ could exist, being stable, until 300 GPa.

In 2015, Drozdov et al. [22] further confirmed a $T_{\mathrm{c}}$ of $203 \mathrm{~K}$ by magnetic susceptibility measurements. In 2016 , Einaga et al. [42] proved the decomposition mechanism of $\mathrm{H}_{2} \mathrm{~S}$ to $\mathrm{H}_{3} \mathrm{~S}+\mathrm{S}$ under pressure and concluded, based on synchrotron X-ray diffraction and resistance measurements, that the highest critical temperature corresponds to the $\mathrm{Im}$ $3 m$ phase. In the same year, Troyan et al. [43] placed a thin ${ }^{119} \mathrm{Sn}$ film inside the $\mathrm{H}_{2} \mathrm{~S}$ sample and confirmed the superconducting state up to $140 \mathrm{~K}$ at $150 \mathrm{GPa}$. Based on combined experimental studies with firstprinciples theoretical 

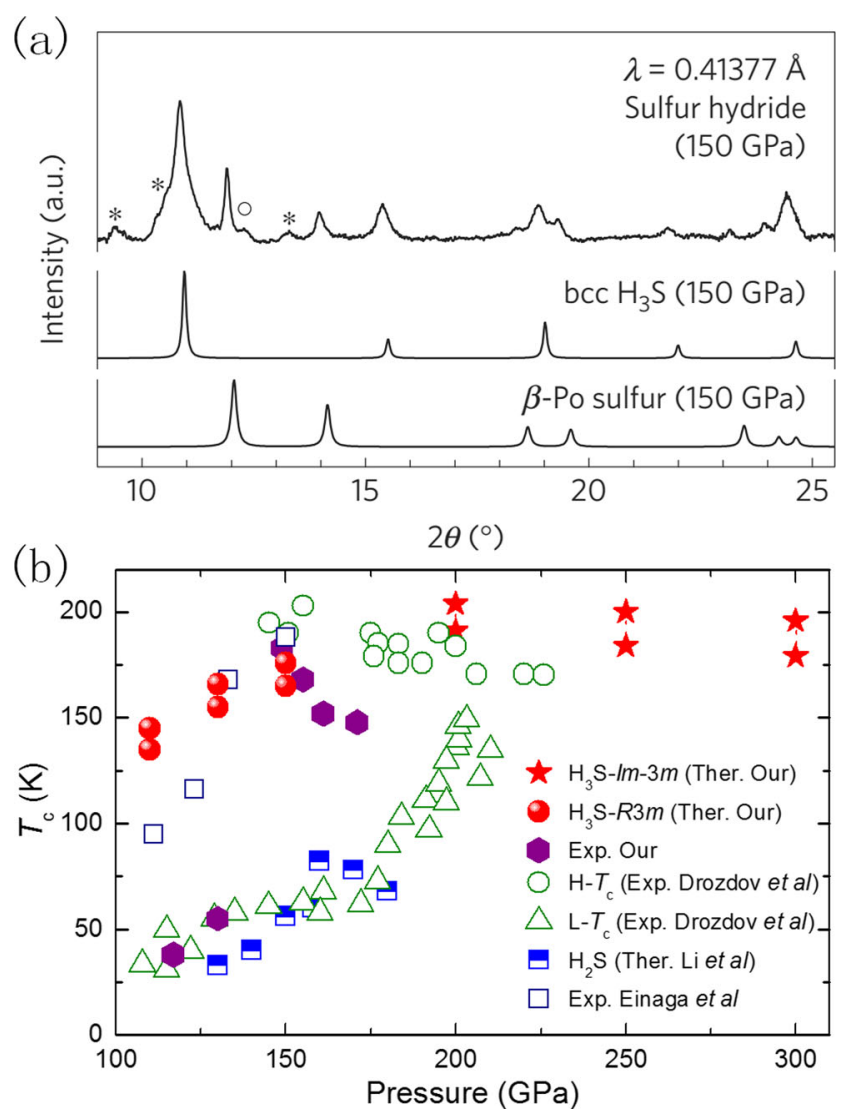

Fig. 3 a XRD patterns of sulfur hydride at $150 \mathrm{GPa}$ [42]. b Pressure dependence of superconducting transition temperature $\left(T_{\mathrm{c}}\right)$ of sulfur hydride by theoretical prediction and experimental measurement

structure searches, Goncharov et al. [44] concluded that $R 3 m$ or $I m-3 m$ phases of $\mathrm{H}_{3} \mathrm{~S}$ were responsible for high $T_{\mathrm{c}}$ superconductivity. On the basis of theoretical studies, Huang et al. [45] also performed in situ alternating current magnetic susceptibility measurements on compressed $\mathrm{H}_{2} \mathrm{~S}$ under high pressures. It was shown that superconductivity suddenly appears at $117 \mathrm{GPa}$ and that $T_{\mathrm{c}}$ reaches $183 \mathrm{~K}$ at $149 \mathrm{GPa}$ before decreasing monotonically with a further increase in pressure. In addition, Goncharov et al. [46] used the directly heated sulfur in hydrogen at various pressures to determine the most stable structures of pure $\mathrm{H}_{3} \mathrm{~S}$. They found that the $C c c m$ and $\operatorname{Im} 3 m \mathrm{H}_{3} \mathrm{~S}$ are the reaction products at $50 \mathrm{GPa}$ and $140 \mathrm{GPa}$, respectively. They also found that $I m-3 m \mathrm{H}_{3} \mathrm{~S}$ transformed to $R 3 m \mathrm{H}_{3} \mathrm{~S}$ on pressure release below $140 \mathrm{GPa}$. Mazin [47] emphasizes in the Nature News \& Views "this is the first time that a previously unknown material predicted to be a hightemperature superconductor has been experimentally confirmed to be one".

Ever since the recorded high $T_{\mathrm{c}}$ is discovered, the theoretical physicist has been working hand in hand with the experimental physicist endeavoring to explore the structure, vibrational properties, conductivity, optical properties, and other properties of $\mathrm{H}_{3} \mathrm{~S}$. Considering the anharmonic approximation of phonon, the electron-phonon coupling is reduced by $30 \%$, and $T_{\mathrm{c}}$ is inhibited [48]. By means of ab initio calculations with the PBE0 hybrid exchangecorrelation approximation, the calculated $T_{\mathrm{c}}$ is enhanced by $25 \%$ [49]. Considering anti-adiabatic approximation [50], superconducting properties of $I m-3 m-\mathrm{H}_{3} \mathrm{~S}$ phase are influenced obviously by pockets near Fermi surface, which are characterized by large density of states [51, 52]. However, Gor'kov and Kresin discussed on non-adiabaticity and thought that these pockets just lead to an appearance of the two-gap picture $[53,54]$. Considering zero vibration effect and anharmonic effect, Sano et al. [52] think the $T_{\mathrm{c}}$ may be changes in 10 30 K. Nicol and Carbotte [55] believed that the highpurity sample may further improve $T_{\mathrm{c}}$ value, and Chu [56] considered that the rational synthesis approach may further improve $T_{\mathrm{c}}$ value from the angle of chemical synthesis. Considering the quantum nature of the proton, Errea et al. [57] predicted that the $R 3 \mathrm{~m}$ phase transforms to the $I m-3 m$ phase at $103 \mathrm{GPa}$ and $I m-3 m-\mathrm{H}_{3} \mathrm{~S}$ dominated the pressure range within which the high $T_{\mathrm{c}}$ was measured. Independently, Yuan et al. [58] come to similar conclusions by ab initio path integral molecular dynamics (PIMD) and speculated that more accurate theoretical methods on the electronic structure level can improve the agreement between theory and experiment. Papaconstantopoulos et al. [59] treated the total $\lambda$ as $\lambda=3 \lambda_{\mathrm{H}}+\lambda_{\mathrm{S}}$ and found that $T_{\mathrm{c}}$ only decreased $19 \mathrm{~K}$ when the contribution of $\mathrm{S}$ to electron-phonon coupling is ignored. Thus, they thought that $I m-3 m-\mathrm{H}_{3} \mathrm{~S}$ could be regarded as a high $T_{\mathrm{c}}$ phase of $\mathrm{H}$ atoms. Tanaka et al. [60] summarize that the metallic- $\mathrm{H}$ bent vibrations are most effective in enhancing $T_{\mathrm{c}}$. And this vibration profile is mainly related to the $\mathrm{H}$ framework of $\mathrm{Im}$ $3 m-\mathrm{H}_{3} \mathrm{~S}$. Akashi et al. [61] have also found that van-Hove singularity structures were slowly generated as the amount of $I m-3 m-\mathrm{H}_{3} \mathrm{~S}$ units increased in the transitional structure during the transition phase. Bianconi and Jarlborg [62, 63] and Jarlborg and Bianconi [64] found that the change of $T_{\mathrm{c}}$ with pressure may correspond to Lifshitz transition, and zeropoint vibration energy also has a negligible impact.

As an important means to achieve or affect the superconductivity of materials, doping has been widely used in a variety of superconducting system research $[65,66]$. Thus, combining with the supercell and the virtual crystal approximation, many theoretical physicists study the effect of light elements doping in superconducting $\mathrm{H}_{3} \mathrm{~S}$ at high pressure by means of the firstprinciples calculation based on density functional theory. Heil and Boeri [67] used chalcogen elements as doping elements and studied the superconductivity of $I m-3 m-\mathrm{H}_{3} \mathrm{X}$ at the same pressure with that of $\mathrm{H}_{3} \mathrm{~S}(200$ 
GPa). After comparing a series of ratios, it is considered that the $T_{\mathrm{c}}$ of $\mathrm{H}_{3} \mathrm{O}_{0.5} \mathrm{~S}_{0.5}$ is further improved compared to $\mathrm{H}_{3} \mathrm{~S}$ when the system satisfies the dynamic stability. However, Ge et al. [68] suggested that the addition of the chalcogen element reduced the electron density of the system and reduced the covalent metal properties of the system and therefore did not improve the $T_{\mathrm{c}}$ value. At the same time, they found that $T_{\mathrm{c}}$ increases with the increase of phosphorus concentration and the $T_{\mathrm{c}}$ value decreases when the doping ratio is around $10 \%$. Further studies show that the $T_{\mathrm{c}}$ value of $\mathrm{H}_{3} \mathrm{~S}_{0.925} \mathrm{P}_{0.075}$ at $250 \mathrm{GPa}$ can be as high as $280 \mathrm{~K}$ and the $T_{\mathrm{c}}$ value of $\mathrm{H}_{3} \mathrm{~S}_{0.96} \mathrm{Si}_{0.04}$ at $250 \mathrm{GPa}$ was $274 \mathrm{~K}$. It is obviously that by investigating the interactions between doped and host atoms, electronic band structure, phonon vibration frequency, the electron-phonon interaction of the newly doped systems and the effect of light elements doping in superconducting $\mathrm{H}_{3} \mathrm{~S}$ at high pressure, one may find an efficient way to improve the superconducting transition temperature or reduce the synthetized pressure of $\mathrm{H}_{3} \mathrm{~S}$.

Combining experiments and ab initio calculations [44, 69], researchers proposed many other sulfur hydrides for low- $T_{\mathrm{c}}$ phase, such as $\mathrm{HS}_{2}, \mathrm{H}_{2} \mathrm{~S}_{3}, \mathrm{H}_{3} \mathrm{~S}_{2}, \mathrm{H}_{3} \mathrm{~S}_{5}, \mathrm{H}_{4} \mathrm{~S}_{3}, \mathrm{H}_{5} \mathrm{~S}_{8}$, $\mathrm{H}_{5} \mathrm{~S}_{2}$ and the Magnéli phases. The estimated values of $T_{\mathrm{c}}$ for $\mathrm{H}_{2} \mathrm{~S}$ [39] and $\mathrm{H}_{5} \mathrm{~S}_{2}$ [70] are in good agreement with experimental data obtained below $180 \mathrm{GPa}$. At the same time, multiple $\mathrm{H}_{2} \mathrm{~S}$ decomposition and $\mathrm{H}_{3}$ Sgenerated paths were pointed out, for example $3 \mathrm{H}_{2} \mathrm{~S} \rightarrow 2 \mathrm{H}_{3} \mathrm{~S}+\mathrm{S}$ [40], $8 \mathrm{H}_{2} \mathrm{~S} \rightarrow \mathrm{S}+4 \mathrm{H}_{3} \mathrm{~S}+\mathrm{H}_{4} \mathrm{~S}_{3}$ [69], $2 \mathrm{H}_{2} \mathrm{~S}+\mathrm{H}_{2} \rightarrow 2 \mathrm{H}_{3} \mathrm{~S}$ [21] and so on. In order to clarify the decomposition of $\mathrm{H}_{2} \mathrm{~S}$, further experimental and theoretical studies of $\mathrm{H}_{2} \mathrm{~S}$ at high pressure are still greatly demanded.

\section{Superconductivity in Lanthanum Hydrides}

Recently, novel H-rich clathrate structures have been theoretically predicted with high $T_{\mathrm{c}}$ values, $\mathrm{YH}_{6}(264 \mathrm{~K}$ at $120 \mathrm{GPa}$ ) [71], $\mathrm{ScH}_{9}$ (nearly $200 \mathrm{~K}$ at $400 \mathrm{GPa}$ ) [72, 73], and $\mathrm{LaH}_{10}(286 \mathrm{~K}$ at $210 \mathrm{GPa})[72,74]$. The crystal structures and clathrate cages are shown in Fig. 4. For $\mathrm{YH}_{6}$, it crystallizes the cubic $I m-3 m$ structure, in which six $\mathrm{H}$ squares and eight $\mathrm{H}$ hexagons form to the $\mathrm{H}$ cage and $\mathrm{Y}$ atom at the cage center (Fig. $4 \mathrm{a}$ ). $\mathrm{ScH}_{9}$ adopts a hexagonal structure with the space group $P 6_{3} / m m c$, in which $\mathrm{H}$ cage consists of six irregular squares, six pentagons, and six hexagons (Fig. 4b).

In May 2017, Liu et al. [74] theoretically predicted, using the CALYPSO code, that $\mathrm{LaH}_{3-5}, \mathrm{LaH}_{8}$, and $\mathrm{LaH}_{10}$ were stable at $150 \mathrm{GPa}$. $\mathrm{LaH}_{8}$ was comprised of an extended hydrogenic lattice (space group $C 2 / m$ ) with the $\mathrm{H}-\mathrm{H}$ distance of $1.02 \AA$ at $300 \mathrm{GPa}$. The calculated electroncoupling parameter $(\lambda)$ and superconducting transition temperature $\left(T_{\mathrm{c}}\right)$ reached $1.12 \mathrm{~K}$ and $114-131 \mathrm{~K}$ with $\mu^{*}=0.1-0.13$, respectively. $\mathrm{LaH}_{10}$ shared a sodalite-like structure with the space group $\mathrm{Fm}-3 m$ (isotypic to the $\mathrm{YH}_{10}$ phase), wherein six $\mathrm{H}$ squares and $12 \mathrm{H}$ hexagons form to the $\mathrm{H}$ cage (Fig. 4c). The computed EPC was approximately 3.41 , and the estimated $T_{\mathrm{c}}$ from the Eliashberg equations was $274-286 \mathrm{~K}$ at $210 \mathrm{GPa}$. Furthermore, the calculated results showed that the values of $\lambda$ and $T_{\mathrm{c}}$ decreased with increasing pressure. In September 2017, Peng et al. [72] produced a large work on rare earth hydride RE-H system $(\mathrm{RE}=\mathrm{Sc}, \mathrm{Y}, \mathrm{La}, \mathrm{Ce}, \mathrm{Pr}$, etc. $)$ by means of CALYPSO and AIRSS codes. They studied the stability of $\mathrm{LaH}_{n}(n=2-12)$
Fig. 4 Clathrate structures of $\mathbf{a}$ $\mathrm{YH}_{6}, \mathbf{b} \mathrm{ScH}_{9}$, and $\mathbf{c} \mathrm{LaH}_{10}$

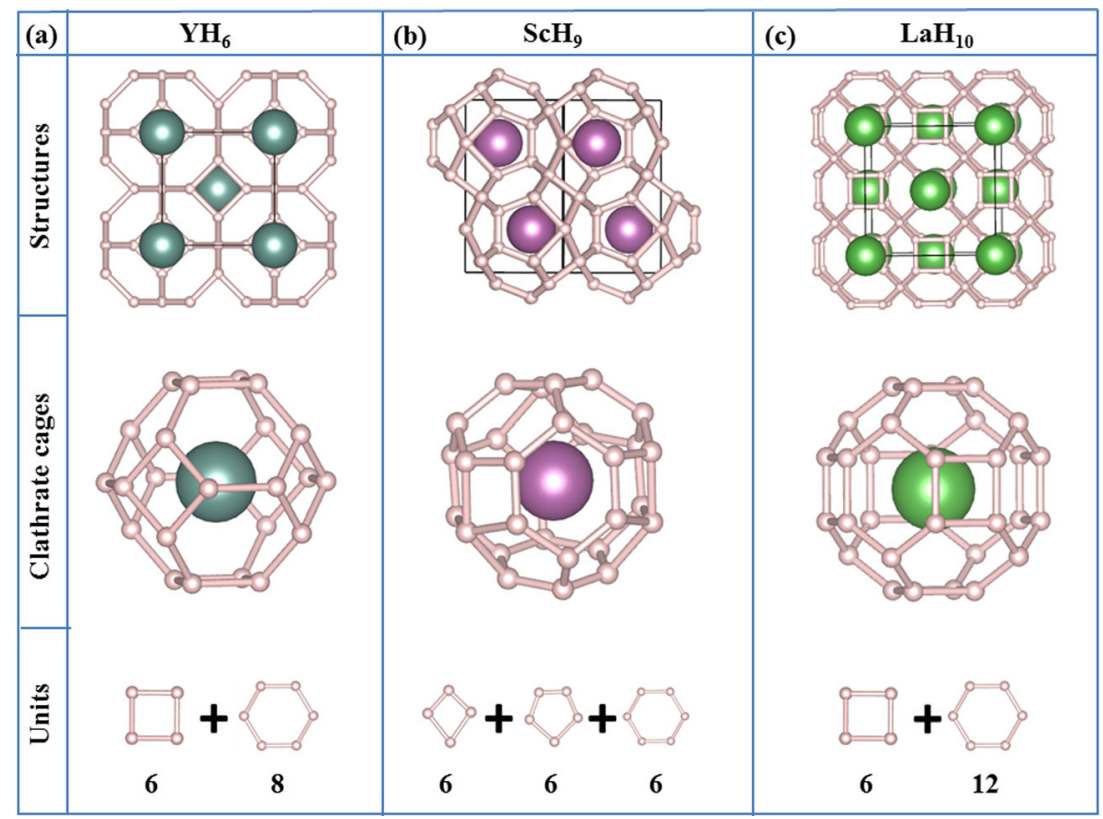


in the pressure range of 50-200 GPa and found $\mathrm{LaH}_{5-7}$ and $\mathrm{LaH}_{9}$ can be stable at different pressures. They also found the same phase $F m-3 m$ in $\mathrm{LaH}_{10}$ and predicted, using $\mu^{*}=$ 0.1 , that its $T_{\mathrm{c}}$ can reach $288 \mathrm{~K}$ at $200 \mathrm{GPa}$.

Most recently, Somayazulu et al. [75] reported that lanthanum superhydride, with X-ray measurements consistent with the theoretically predicted structure for $\mathrm{LaH}_{10}$, can be synthesized at $170 \mathrm{GPa}$ upon heating to $\sim 1000 \mathrm{~K}$, with the use of ammonia borane as the hydrogen source. The transport measurements displayed a significant drop in resistivity on cooling at $260 \mathrm{~K}$ and pressures of 190 GPa [75]. Meanwhile, Drozdov et al. [76] also synthesized lanthanum hydride $\left(\mathrm{LaH}_{x}\right)$ through laser heating of lanthanum in hydrogen at the same pressure (170 GPa), though a slightly different value of $T_{\mathrm{c}}$. In the experiment, when pressure decreased to $150 \mathrm{GPa}, T_{\mathrm{c}}$ increased from 209 to $215 \mathrm{~K}$. It is exciting that two groups separately discovered the evidence for superconductivity in the La-H system at high pressures. But, for further proofs such as Meissner effect measurements, isotope effect measurements are also needed. In addition, both paper [72, 74] have reported that $\mathrm{YH}_{10}$ has same structure as $F m-3 m \mathrm{LaH}_{10}$; they are predicted to be room-temperature superconductors (above $300 \mathrm{~K}$ ) above $250 \mathrm{GPa}$. And, we hope it can be observed experimentally in the future.

\section{Summary and Outlook}

In this article, we have described recent ab initio calculations of $T_{\mathrm{c}}$ in hydrogen-based superconductors, especially sulfur hydrides and lanthanum hydrides. Theoretical prediction and experimental confirmations for $\mathrm{H}_{3} \mathrm{~S}$ and $\mathrm{LaH}_{10}$ with high $T_{\mathrm{c}}$ above $200 \mathrm{~K}$ accelerate the arrival of roomtemperature superconductors which are waving hands to us. The next challenge is to find the paths to reduce pressure for these hydrogen-based superconductors. Ab initio calculation power has been verified, and more high $T_{\mathrm{c}}$ superconductors can be expected to be found in hydrogen-rich materials at much lower pressure in the future, for example the ternary hydrides, doping some light elements etc.

Acknowledgements We thank Professor Vladimir Kresin for many interesting and stimulating discussions.

Funding Information This work was supported by the National Key R\&D Program of China (No. 2018YFA0305900), National Natural Science Foundation of China (Nos. 11674122, 51632002, 51572108, 91745203, 11634004, 11504127, 11574109, 11704143, and 11404134), National Key Research and Development Program of China (2016YFB0201204), Jilin Provincial Science and Technology Development Project of China (20170520116JH), Program for Changjiang Scholars and Innovative Research Team in University (No. IRT_15R23), and National Found for Fostering Talents of Basic Science (No. J1103202).
Open Access This article is distributed under the terms of the Creative Commons Attribution 4.0 International License (http://creativecommons. org/licenses/by/4.0/), which permits unrestricted use, distribution, and reproduction in any medium, provided you give appropriate credit to the original author(s) and the source, provide a link to the Creative Commons license, and indicate if changes were made.

\section{References}

1. Onnes, H.K.: Commun. Phys. Lab. Univ. Leiden 12, 1 (1911)

2. Testardi, L.R.: Rev. Mod. Phys. 47, 637 (1975)

3. Bednorz, J.G., Müller, K.A.: Z. Physik. B 64, 189 (1986)

4. Schilling, A., Cantoni, M., Guo, J.D., Ott, H.R.: Nature 363, 56 (1993)

5. Gao, L., Xue, Y.Y., Chen, F., Xiong, Q., Meng, R.L., Ramirez, D., Chu, C.W., Eggert, J.H., Mao, H.K.: Phys. Rev. B 50, 4260 (1994)

6. Wu, G., Xie, Y., Chen, H., Zhong, M., Liu, R., Shi, B., Li, Q., Wang, X., Wu, T., Yan, Y.: J. Phys: Condens. Matter 21, 142203 (2009)

7. Ge, J.-F., Liu, Z.-L., Liu, C., Gao, C.-L., Qian, D., Xue, Q.-K., Liu, Y., Jia, J.-F.: Nat. Mater. 14, 285 (2015)

8. Nagamatsu, J., Nakagawa, N., Muranaka, T., Zenitani, Y., Akimitsu, J.: Nature 410, 63 (2001)

9. Allen, P.B.: Phys. Rev. B 6, 2577 (1972)

10. Allen, P.B., Silberglitt, R.: Phys. Rev. B 9, 4733 (1974)

11. Allen, P.B., Dynes, R.C.: Phys. Rev. B 12, 905 (1975)

12. McMillan, W.L.: Phys. Rev. 167, 331 (1968)

13. Goedecker, S.: J. Chem. Phys. 120, 9911 (2004)

14. Oganov, A.R., Glass, C.W.: J. Chem. Phys. 124, 244704 (2006)

15. Falls, Z., Lonie, D.C., Avery, P., Shamp, A., Zurek, E.: Comput. Phys. Commun. 199, 178 (2016)

16. Pickard, C.J., Needs, R.J.: J. Phys.: Condens. Matter 23, 053201 (2011)

17. Wang, Y., Lv, J., Zhu, L., Ma, Y.: Comput. Phys. Commun. 183, 2063 (2012)

18. Dias, R.P., Silvera, I.F.: Science 355, 715 (2017)

19. Service, R.: Science 355, 332 (2017)

20. Ashcroft, N.: Phys. Rev. Lett. 92, 187002 (2004)

21. Duan, D., Liu, Y., Tian, F., Li, D., Huang, X., Zhao, Z., Yu, H., Liu, B., Tian, W., Cui, T.: Scientific Reports 4, 6968 (2014)

22. Drozdov, A., Eremets, M., Troyan, I., Ksenofontov, V., Shylin, S.: Nature 525, 73 (2015)

23. Chen, X.-J., Struzhkin, V.V., Song, Y., Goncharov, A.F., Ahart, M., Liu, Z., Mao, H.-K., Hemley, R.J.: Proc. Natl. Acad. Sci. USA 105, 20 (2008)

24. Eremets, M., Trojan, I., Medvedev, S., Tse, J., Yao, Y.: Science 319, 1506 (2008)

25. Chen, X.-J., Wang, J.-L., Struzhkin, V.V., Mao, H.-K., Hemley, R.J., Lin, H.-Q.: Phys. Rev. Lett. 101, 077002 (2008)

26. Martinez-Canales, M., Oganov, A.R., Ma, Y., Yan, Y., Lyakhov, A.O., Bergara, A.: Phys. Rev. Lett. 102, 087005 (2009)

27. Gao, G., Oganov, A.R., Bergara, A., Martinez-Canales, M., Cui, T., Iitaka, T., Ma, Y., Zou, G.: Phys. Rev. Lett. 101, 107002 (2008)

28. Zhang, C., Chen, X.-J., Li, Y.-L., Struzhkin, V.V., Mao, H.-K., Zhang, R.-Q., Lin, H.-Q.: Europhys. Lett. 90, 66006 (2010)

29. Tse, J., Yao, Y., Tanaka, K.: Phys. Rev. Lett. 98, 117004 (2007)

30. Gao, G., Oganov, A.R., Li, P., Li, Z., Wang, H., Cui, T., Ma, Y., Bergara, A., Lyakhov, A.O., Iitaka, T.: Proc. Natl. Acad. Sci. USA 107, 1317 (2010)

31. Jin, X., Meng, X., He, Z., Ma, Y., Liu, B., Cui, T., Zou, G., Mao, H.-K.: Proc. Natl. Acad. Sci. USA 107, 9969 (2010)

32. Abe, K., Ashcroft, N.: Phys. Rev. B 84, 104118 (2011) 
33. Chen, C., Tian, F., Wang, L., Duan, D., Cui, T., Liu, B., Zou, G.: J. Phys. Condens. Matter 22, 225401 (2010)

34. Zhong, G., Zhang, C., Wu, G., Song, J., Liu, Z., Yang, C.: Phys. B: Condens. Matter 410, 90 (2013)

35. Zhou, D., Jin, X., Meng, X., Bao, G., Ma, Y., Liu, B., Cui, T.: Phys. Rev. B 86, 014118 (2012)

36. Wang, H., John, S.T., Tanaka, K., Iitaka, T., Ma, Y.: Proc. Natl. Acad. Sci. USA 109, 6463 (2012)

37. Strobel, T.A., Ganesh, P., Somayazulu, M., Kent, P., Hemley, R.J.: Phys. Rev. Lett. 107, 255503 (2011)

38. Drozdov, A., Eremets, M., Troyan, I.: arXiv:1412.0460 (2014)

39. Li, Y., Hao, J., Liu, H., Li, Y., Ma, Y.: J. Chem. Phys. 140, 174712 (2014)

40. Duan, D., Huang, X., Tian, F., Li, D., Yu, H., Liu, Y., Ma, Y., Liu, B., Cui, T.: Phys. Rev. B 91, 180502 (2015)

41. Bernstein, N., Hellberg, C.S., Johannes, M., Mazin, I., Mehl, M.: Phys. Rev. B 91, 060511 (2015)

42. Einaga, M., Sakata, M., Ishikawa, T., Shimizu, K., Eremets, M.I., Drozdov, A.P., Troyan, I.A., Hirao, N., Ohishi, Y.: Nat. Phys. 12, 835 (2016)

43. Troyan, I., Gavriliuk, A., Rüffer, R., Chumakov, A., Mironovich, A., Lyubutin, I., Perekalin, D., Drozdov, A.P., Eremets, M.I.: Science 351, 1303 (2016)

44. Goncharov, A.F., Lobanov, S.S., Kruglov, I., Zhao, X.-M., Chen, X.-J., Oganov, A.R., Konôpková, Z., Prakapenka, V.B.: Phys. Rev. B 93, 174105 (2016)

45. Huang, X., Wang, X., Duan, D., Sundqvist, B., Li, X., Huang, Y., Li, F., Zhou, Q., Liu, B., Cui, T.: arXiv:1610.02630 (2016)

46. Goncharov, A.F., Lobanov, S.S., Prakapenka, V.B., Greenberg, E.: Phys. Rev. B 95, 140101 (2017)

47. Mazin, I.I.: Nature 525, 40 (2015)

48. Errea, I., Calandra, M., Pickard, C.J., Nelson, J., Needs, R.J., Li, Y., Liu, H., Zhang, Y., Ma, Y., Mauri, F.: Phys. Rev. Lett. 114, 157004 (2015)

49. Komelj, M., Krakauer, H.: Phys. Rev. B 92, 205125 (2015)

50. Baňacký, P.: Results Phys. 6, 1 (2016)

51. Quan, Y., Pickett, W.E.: Phys. Rev. B 93, 104526 (2016)

52. Sano, W., Koretsune, T., Tadano, T., Akashi, R., Arita, R.: Phys. Rev. B 93, 094525 (2016)
53. Gor'kov, L.P., Kresin, V.Z.: Scientific Reports 6, 25608 (2016)

54. Gor'kov, L.P., Kresin, V.Z.: Rev. Mod. Phys. 90, 011001 (2018)

55. Nicol, E.J., Carbotte, J.P.: Phys. Rev. B 91, 220507 (2015)

56. Chu, K.-H.R.: Mater. Lett. 172, 188 (2016)

57. Errea, I., Calandra, M., Pickard, C.J., Nelson, J.R., Needs, R.J., Li, Y., Liu, H., Zhang, Y., Ma, Y., Mauri, F.: Nature 532, 81 (2016)

58. Yuan, Y., Feng, Y., Bian, L., Zhang, D.B., Li, X.Z. (2016)

59. Papaconstantopoulos, D.A., Klein, B.M., Mehl, M.J., Pickett, W.E.: Phys. Rev. B 91, 184511 (2015)

60. Tanaka, K., Tse, J., Liu, H.: Phys. Rev. B 96, 100502 (2017)

61. Akashi, R., Sano, W., Arita, R., Tsuneyuki, S.: Phys. Rev. Lett. 117, 075503 (2016)

62. Bianconi, A., Jarlborg, T.: Europhys. Lett. 112, 37001 (2015)

63. Bianconi, A., Jarlborg, T.: Nov. Supercond. Mater. 1, 37 (2015)

64. Jarlborg, T., Bianconi, A.: Scientific Reports 6, 24816 (2016)

65. Ekimov, E., Sidorov, V., Bauer, E., Mel'Nik, N., Curro, N., Thompson, J., Stishov, S.: Nature 428, 542 (2004)

66. Takano, Y., Takenouchi, T., Ishii, S., Ueda, S., Okutsu, T., Sakaguchi, I., Umezawa, H., Kawarada, H., Tachiki, M.: Diam. Relat. Mater. 16, 911 (2007)

67. Heil, C., Boeri, L.: Phys. Rev. B 92, 060508 (2015)

68. Ge, Y., Zhang, F., Yao, Y.: Phys. Rev. B 93, 224513 (2016)

69. Li, Y., Wang, L., Liu, H., Zhang, Y., Hao, J., Pickard, C.J., Nelson, J.R., Needs, R.J., Li, W., Huang, Y.: Phys. Rev. B 93, 020103 (2016)

70. Ishikawa, T., Nakanishi, A., Shimizu, K., Katayama-Yoshida, H., Oda, T., Suzuki, N.: Scientific Reports 6, 23160 (2016)

71. Li, Y., Hao, J., Liu, H., Tse, J.S., Wang, Y., Ma, Y.: Sci. Rep. 5, 9948 (2015)

72. Peng, F., Sun, Y., Pickard, C.J., Needs, R.J., Wu, Q., Ma, Y.: Phys. Rev. Lett. 119, 107001 (2017)

73. Ye, X., Hoffmann, R., Ashcroft, N.W.: J. Phys. Chem. C 119, $5614(2015)$

74. Liu, H.Y., Naumov, I.I., Hoffmann, R., Ashcroft, N.W., Hemley, R.J.: Proc. Natl. Acad. Sci. U.S.A. 114, 6990 (2017)

75. Somayazulu, M., Ahart, M., Mishra, A.K., Geballe, Z.M., Baldini, M., Meng, Y., Struzhkin, V.V., Hemley, R.J.: arXiv:1808.07695 (2018)

76. Drozdov, A.P., Minkov, V.S., Besedin, S.P., Kong, P.P., Kuzovnikov, M.A., Knyazev, D.A., Eremets, M.I.: arXiv:1808. 07039 (2018) 